Revista de Investigación Cientiffica y Tecnolo̊gica

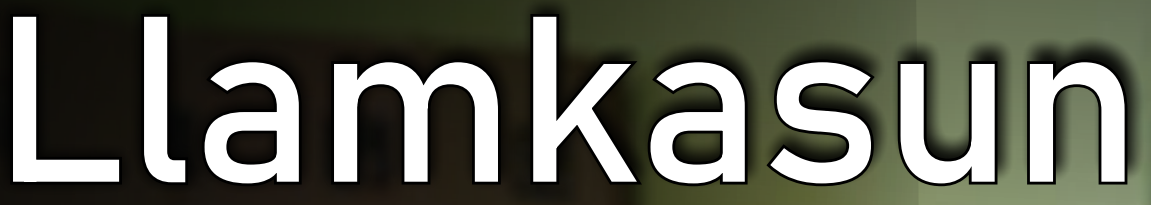

ISSN: 2709-2275

Trabajemos

Vol. 02 Num. 03 - Junio 2021

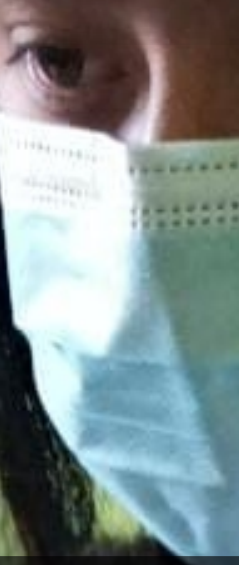

Impacto Psicológico en la población ocasionado por la COVID-19

를 $\quad$ Psychological impact on the population caused by COVID-19

(46) Impacto psicológico na população causado pelo COVID-19

https://doi.org/10.47797/llamkasun.v2i3.59

Edición Especial

COVID - 19

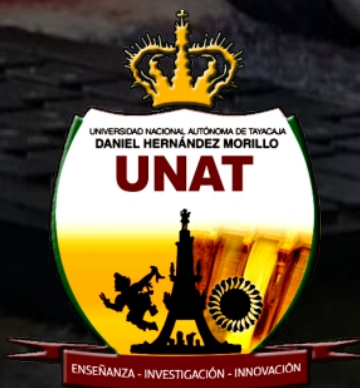

UNIVERSIDAD NACIONAL AUTÓNOMA DE TAYACAJA DANIEL HERNÁNDEZ MORILLO VICEPRESIDENCIA DE INVESTIGACIÓN 


\title{
Impacto Psicológico en la población ocasionado por la COVID-19
}

Psychological impact on the population caused by COVID-19

Impacto psicológico sobre a população causado pela COVID-19

\author{
Jorge Nelson Malpartida Gutiérrez \\ Universidad Nacional Autónoma de Tayacaja Daniel Hernández Morillo \\ Rebeca Mato Rojas \\ Universidad de Huánuco \\ Karina Satalaya Vicente \\ Universidad de Huánuco \\ Anali Marleni Huachaca Pacheco \\ Universidad Nacional Autónoma de Tayacaja Daniel Hernández Morillo \\ Mildred Jénica Ledesma cuadros ORCID \\ Universidad César Vallejo \\ Jorge Rafael Diaz Dumont \\ Universidad Nacional Autónoma de Tayacaja Daniel Hernández Morillo
}

\section{RESUMEN}

La pandemia producida por la COVID-19 generó diversos problemas psicológicos en la población (estrés y frustración) como producto del aislamiento social y el confinamiento. El objetivo del presente estudio fue conocer el impacto psicológico de la población ocasionada por la COVID-19. La investigación corresponde a una revisión sistemática, se seleccionó revistas científicas en idioma español e inglés de base de datos: EBSCOhost, PROQUEST, SCIELO, SPRINGER LINK y GOOGLE ACADÉMICO, se tomaron en cuenta criterios para su delimitación las cuales correspondieron entre los años 2020 y 2021. Del análisis realizado, se tomaron 12 artículos que respondían a la pregunta de investigación, concluyendo de esta manera que la COVID-19 trajo consigo efectos negativos en la salud mental de la población.

Palabras clave: COVID-19, salud mental, confinamiento

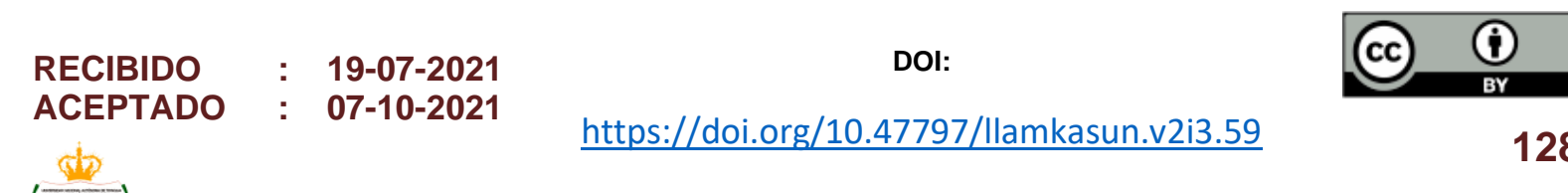




\begin{abstract}
The pandemic caused by COVID-19 generated various psychological problems in the population (stress and frustration) as a result of social isolation and confinement. The objective of this study was to determine the psychological impact of COVID-19 on the population. The research corresponds to a systematic review, scientific journals in Spanish and English language were selected from databases: EBSCOhost, PROQUEST, SCIELO, SPRINGER LINK and GOOGLE ACADEMIC, criteria were taken into account for their delimitation which corresponded between the years 2020 and 2021. From the analysis carried out, 12 articles were taken that responded to the research question, thus concluding that COVID-19 brought about negative effects on the mental health of the population.
\end{abstract}

Keywords: COVID-19, mental health, confinement

\title{
RESUMO
}

A pandemia causada pela COVID-19 gerou vários problemas psicológicos na população (stress e frustração) como resultado do isolamento social e do confinamento. O objectivo deste estudo era determinar o impacto psicológico da COVID-19 sobre a população. A investigação corresponde a uma revisão sistemática, foram seleccionadas revistas científicas em espanhol e inglês a partir de bases de dados: EBSCOhost, PROQUEST, SCIELO, SPRINGER LINK e GOOGLE ACADEMIC, foram tidos em conta critérios para a sua delimitação que corresponderam entre os anos de 2020 e 2021. Da análise efectuada, foram retirados 12 artigos que responderam à questão da investigação, concluindo assim que a COVID-19 trouxe consigo efeitos negativos para a saúde mental da população.

Palavras-chave: COVID-19, saúde mental, confinamento 


\section{INTRODUCCIÓN}

La Organización Mundial de la Salud (OMS) en el mes de enero de 2020, declaró como brote la enfermedad del COVID-19, esta enfermedad de manera rápida se propago en muchos países del mundo; debido a ello, el 11 de marzo de 2020 se le dio la categoría de pandemia.

Muchos países en el mundo tomaron medidas para detener el contagio; entre ellas, el aislamiento total de las personas portadoras del virus y de las que se tiene sospecha de haber contraído el virus. Este proceso se realizó de dos maneras, el aislamiento y las cuarentenas, para así reducir el contacto entre personas y evitar la propagación del virus (Palomino-Oré \& Huarcaya-Victoria, 2020).

La situación pandémica ocasionada por la COVID-19 presenta características que incrementan la presencia del estrés por tratarse de un virus nuevo, generando incertidumbre en la población por no saber cómo manejar la situación (Palomino-Oré \& Huarcaya-Victoria, 2020).

Los seres humanos manifestaron diversas reacciones como respuesta a esta situación de amenaza. Fue inevitable controlar estas reacciones por ser causas externas, como por ejemplo la angustia, el miedo a perder a un ser querido, el trabajo, negocios y el pánico al no saber qué sucederá en el futuro (Alvites-Huamaní, 2020).

Las familias con antecedentes de cuadros de depresión y estrés son más vulnerables a padecer estrés; por lo tanto, se debe tener mayor consideración ya que el 30-40\% de personas afectadas podrían desarrollar un trastorno por estrés postraumático (TEPT) como ocurrió en desastres anteriores. En este sentido las personas de la tercera edad poseen incluso 2,11 mayores probabilidades de adquirir el síntoma de TEPT comparado con adultos jóvenes. (Palomino-Oré \& Huarcaya-Victoria, 2020) Mientras la población se encuentre en cuarentena dentro de su hogar, pueden experimentar dos tipos de reacciones, por ejemplo, algunas personas pueden reaccionar de manera positiva y otras por el contrario, pueden desarrollar estrés, cansancio y otros trastornos de carácter psicológico (Marquina \& JaramilloValverde, 2020).

En los adultos mayores, el efecto del confinamiento fue mayor y estudios recientes confirman que la COVID-19 los ha llevado a un serio desgaste en su salud mental (Broche et al., 2020); existiendo mayor probabilidad de desarrollar cuadros de ansiedad, mal humor, estrés, agitación y retraimiento durante su periodo de cuarentena (Hernández, 2020). 
Actualmente, se requiere de forma urgente, atención oportuna del impacto psicológico que ocasiona el COVID-19 en la población en general (Marquina \& JaramilloValverde, 2020).

El objetivo de este estudio fue conocer el impacto psicológico en la población ocasionado por la COVID-19.

\section{Método de desarrollo}

La presente investigación utilizó la técnica del análisis documental, la cual consistió en revisar la literatura existente. La revisión sistemática se realizó a través de la búsqueda de información en diferentes bases de datos EBSCOhost, PROQUEST, SCIELO, SPRINGER Link y GOOGLE ACADÉMICO.

Este estudio incluyó investigaciones observacionales y revisiones sistemáticas en idiomas español e inglés.

Búsqueda de información:

El inicio de la recopilación de información se realizó empleando las palabras clave: psychological impact, impacto psicológico y COVID-19, con ello se empezó a realizar la búsqueda de artículos científicos en la base de datos: EBSCOHOST, PROQUEST, SCIELO, SPRINGER LINK Y GOOGLE ACADÉMICO.
También se utilizaron operadores booleanos que permitieron conectar de forma lógica las palabras para limitar la búsqueda, considerando dentro de ellos a OR y AND. Los artículos que se seleccionaron pertenecían a los años 2020 - 2021. Asimismo, se consideró criterios delimitantes o filtros, el tipo de fuente que son revistas científicas escritas en inglés. y español.

Se trazó la ruta de búsquedas de acuerdo a las bases de datos identificadas:

EBSCOhost: ("Impacto Psicológico" OR "Psychological Impact") AND ("COVID19”) siendo incluidos artículos comprendidos entre los años 2020 - 2021 tanto en idioma inglés como español.

PROQUEST: (“COVID-19”) AND ("Impacto Psicológico" OR "Psychological Impact") siendo incluidos artículos de revistas científicas comprendidas entre los años 2020 - 2021 tanto en idioma inglés como español.

SCIELO: ("Psychological Impact") AND (“COVID-19") siendo incluidos artículos de revistas científicas comprendidas entre los años 2020 - 2021 tanto en idioma inglés como español.

SPRINGER LINK: ("Psychological Impact") AND (“COVID-19”) siendo incluidos artículos de revistas científicas 
comprendidas entre los años 2020 - 2021

tanto en idioma inglés.

\section{Criterios de inclusión y exclusión}

\section{Criterios de inclusión:}

Se seleccionó por año de publicación entre 2020-2021, solo artículos científicos, idioma español e inglés y estudios que respondan a la pregunta de investigación.

\section{Criterios de exclusión:}

Fueron excluidos los artículos científicos duplicados que se encontraron en las bases consultadas, manuscritos que no tenían carácter de artículo científico, estudios que no aparecen completos e investigaciones que presentan acceso restringido.

\section{Proceso de selección de artículos}

Se encontraron en total 7879 artículos comprendidos entre los años 2020 - 2021, estos se organizaron de la siguiente forma:

EBSCOhost: 2214 artículos

PROQUEST: 49 artículos

SCIELO: 38 artículos

SPRINGER LINK: 2878 artículos

GOOGLE ACADÉMICO: 2700 artículos

Es así que, en EBSCOhost se realizó el filtrado por año de publicación entre 20202021 quedando 2200 artículos, luego se tomó la información evaluada por expertos quedando 89 artículos dentro de los cuales se eligió 1 artículo.

En PROQUEST luego de realizar el filtrado, teniendo en cuenta el año de publicación comprendido entre 2020-2021, dio como resultado 49 artículos de los cuales se citó a 1 en el presente artículo.

En SCIELO se realizó el filtrado teniendo en cuenta el año de antigüedad 2020-2021 en el idioma inglés y español quedando 38 referencias, tomándose 4 en la elaboración del presente artículo.

En SPRINGER Link se realizó el filtrado considerando los años 2020 - 2021, de los cuales quedaron 2878 referencias habiendo sido seleccionados solo artículos científicos en inglés quedando 2017 artículos de los cuales se tomaron 3 de ellos.

En GOOGLE ACADÉMICO se efectúo el filtrado por año de publicación entre 20202021 quedando 2700 referencias dentro de los cuales finalmente se escogió 3 artículos.

\section{Tabla 1}

Selección de artículos después del descarte

\begin{tabular}{lccc}
\hline \multicolumn{1}{c}{ Base de datos } & $\begin{array}{c}\text { Número } \\
\text { de } \\
\text { artículos }\end{array}$ & $\begin{array}{c}\text { Número de } \\
\text { artículos } \\
\text { descartados }\end{array}$ & $\begin{array}{c}\text { Número de } \\
\text { artículos } \\
\text { elegidos }\end{array}$ \\
\hline EBSCO host & 2214 & 2213 & 1 \\
ProQuest & 49 & 48 & 1 \\
Scielo & 38 & 33 & 4 \\
Springer Link & 2878 & 2875 & 3 \\
Google Académico & 2700 & 2698 & 3 \\
$\quad$ TOTAL & & & 12 \\
\hline
\end{tabular}


De acuerdo con los hallazgos se han seleccionado 12 artículos los cuales tienen referencia al tema de investigación respecto al impacto psicológico en la población ocasionado por el COVID-19.

\section{Desarrollo}

A fines del año 2019 brotó una nueva enfermedad en Wuhan, China provocada por un virus denominado SARS-CoV-2 o COVID-19. El 11 de marzo de 2020 fue declarado por la Organización Mundial de la Salud como pandemia global, debido a su propagación a nivel mundial, afectando a más de 100 países, con más de 100.000 casos provocados por este nuevo virus (Sandín et al., 2020).

La COVID-19 es una enfermedad producida por una cepa nueva del coronavirus que genera el síndrome respiratorio agudo (SARS). Se reporta que este virus tiene un $2.3 \%$ de letalidad siendo así superior a la de la influenza y más contagiosa que el SARS (Marquina \& Jaramillo-Valverde, 2020). La transmisión del virus se da entre una persona infectada a una persona sana como también a través de objetos contaminados. El tiempo de incubación es de 5 a 14 días, durante este tiempo las personas diagnosticadas con esta enfermedad deben guardar distanciamiento social y aislamiento. Como consecuencia de esta enfermedad, muchos países en el mundo se vieron forzados al cierre de los negocios y/o empresas o cualquier otro lugar donde pudiera haber contacto cercano y permanezcan en cuarentena porque al hablar, toser o estornudar se emergen fluidos que aumentan la probabilidad del contagio (Alvites-Huamaní, 2020).

\section{Impacto psicológico en la población}

La Organización Mundial de la Salud (OMS) declaró que el virus de la COVID19 y el confinamiento traen consigo fuertes impactos psicológicos en las sociedades, producto del aislamiento y distanciamiento social, cierre de establecimientos, entre otros; los cuales afectan la salud mental de la población, informó el director regional de la OMS para Europa, Hans Kluge en una conferencia, donde se ponía en alerta sobre el gran impacto psicológico que ocasionaría la COVID-19, a corto, mediano y largo plazo, manifestándose a través de algunos trastornos psicológicos relacionados con el trauma y el estrés que no debería ser ignorado (Lorenzo et al., 2020).

En un estudio realizado en China a principios de la pandemia, se tomó como 
muestra a 1210 personas y se concluyó que el $13,8 \%$ tuvo una sintomatología depresiva leve; el 12,2\%, moderada; y el $4,3 \%$, grave. En otro estudio realizado en este mismo país, al inicio de la pandemia, se tomó una muestra de 52730 personas en el cual se concluyó que el 35\% presentaron estrés psicológico (Huarcaya-Victoria, 2020)

Asimismo, una encuesta realizada en China durante la pandemia, de 56679 personas presentaron numerosas condiciones psicológicas, entre las cuales se encuentran: depresión $(27,9 \%)$, ansiedad (31,6\%), estrés $(24,4 \%)$ e insomnio $(29,2 \%)$. Además, otra investigación realizada a 4872 chinos de 31 provincias mostró una alta prevalencia de depresión (48,3\%) y ansiedad (22,6\%) (Arafa et al., 2021).

\section{Afectación Psicológica que incidirá en los estados de Ánimo}

Las personas frente a una enfermedad pandémica experimentan una secuencia de actitudes, las cuales buscan protegerlo frente a un acontecimiento de amenaza o estrés, impidiéndoles muchas veces, la posibilidad de mantener el control por tratarse de razones externas, esto se visualiza cuando dan muestras de su malestar, miedo a la perdida de sus familiares, sus puestos laborales, sus actividades comerciales, entre otros (Gallegos de San Vicente et al., 2020).

La salud psicológica de la población se ve cada vez más afectada por la situación pandémica que se vive, llevándoles, en muchos casos, a elevados estados de estrés; generándoles secuelas psicosociales (Hernández, 2020).

\section{La cuarentena y sus efectos en la salud mental}

Aunque el distanciamiento social es un mecanismo preventivo primordial en tiempos de pandemia, uno de los efectos principales es que promueve sentimientos de soledad, aumentando la vulnerabilidad de quienes ya padecen un trastorno psiquiátrico (Shah et al., 2021). Tanto la incertidumbre como el confinamiento son detonantes de problemas psicológicos en la población, los cuales generan deterioro de la salud mental.

La incertidumbre y el aislamiento se encuentran relacionados con deficiencias en la salud mental de los seres humanos debido a que desencadenan graves problemas, entre ellas la ansiedad, estrés y depresión (Shah et al., 2021).

En este sentido, uno de los grupos más vulnerables son los ancianos (Grolli et al., 2021). Por lo cual se necesita realizar un 


\section{REFERENCIAS BIBLIOGRÁFICAS}

ya que el virus y los factores estresantes relacionados con el aislamiento social pueden agudizar los síntomas de los trastornos asociados a la edad (Grolli et al., 2021).

\section{CONCLUSIONES}

Muchos países en el mundo al verse afectados a causa de la COVID-19 estuvieron en la obligación de tomar medidas inmediatas para frenar el contagio; entre ellas encontramos: el cierre total de los establecimientos que no proveyeran productos de primera necesidad, cumplimiento de la orden de confinamiento y aislamiento de las personas infectadas y a quienes se tuviera la sospecha de haberse infectado.

Todo este acontecimiento producido por la COVID-19, al igual que las acciones asumidas por los gobiernos se relacionaron con problemas de salud mental y en algunas personas empeoró, como en los adultos mayores otro grupo vulnerable fueron aquellas personas que padecían enfermedades preexistentes y por causa de las largas cuarentenas su problema se agudizó, ya que no podían desarrollar sus actividades habituales, provocándoles cuadros de estrés, depresión y ansiedad.

Alvites-Huamaní, C. G. (2020). COVID19: pandemia que impacta en los estados de ánimo. CienciAmérica, $9(2)$, 354. https://doi.org/10.33210/ca.v9i2.327

Arafa, A., Mohamed, A., Saleh, L., \& Senosy, S. (2021). Psychological Impacts of the COVID-19 Pandemic on the Public in Egypt. Community Mental Health Journal, 57(1), 64-69. https://doi.org/10.1007/s10597-02000701-9

Broche, Y., Fernández, E., \& Reyes, D. (2020). Consecuencias psicológicas de la cuarentena y el aislamiento social durante la pandemia de COVID-19. Revista Cubana de Salud Pública 46, 1-14. http://scielo.sld.cu/scielo.php?script=s ci_arttext\&pid=S0864-

34662020000500007

Gallegos de San Vicente, M., O., Zalaquett, C., Luna, S. E.; Mazo, R., Ortiz, B.; Penagos, J. C., Portillo, N., Torres, I., Urzúa, A., Morgan, M., Polanco, F. A., Florez, A. M. \& Lopes, R (2020). Cómo afrontar la pandemia del Coronavirus (Covid-19) en las 
Américas: recomendaciones y líneas de acción sobre salud mental. Revista Interamericana de Psicología, 54, 1; 4. http://hdl.handle.net/11336/109317

Grolli, R. E., Mingoti, M. E. D., Bertollo, A. G., Luzardo, A. R., Quevedo, J., Réus, G. Z., \& Ignácio, Z. M. (2021). Impact of COVID-19 in the Mental Health in Elderly: Psychological and Biological Updates. Molecular Neurobiology, $58(5)$, 1905-1916. https://doi.org/10.1007/s12035-02002249-x

Hernández, J. (2020). Impacto de la COVID-19 sobre la salud mental de las personas. Medicentro Electrónica, 24(3), 578-594. http://scielo.sld.cu/scielo.php?script=s ci_arttext\&pid=S102930432020000300578

Huarcaya-Victoria, J. (2020). Consideraciones sobre la salud mental en la pandemia de COVID-19. Revista Peruana de Medicina Experimental y Salud Pública, 37(2), 327-334. https://doi.org/10.17843/rpmesp.2020. 370.5419

Lorenzo, A., Díaz, K., \& Zaldívar, D. (2020). La Psicología como Ciencia y Profesión en el Afrontamiento del
COVID-19. Revista Caribeña de Psicología, 4(2), 153-165. https://doi.org/10.37226/rcp.v4i2.481 5

Marquina, R. M. \& Jaramillo-Valverde, L. (2020). El COVID-19: Cuarentena y su Impacto Psicológico en la población. Preprint-Scielo, 1, 1-12. https://doi.org/10.1590/SciELOPreprin ts.452

Palomino-Oré, C., \& Huarcaya-Victoria, J. (2020). Stress disorders caused by quarantine during the COVID-19 pandemic. 20(4). https://doi.org/10.24265/horizmed.20 20.v20n4.10

Sandín, B., Valiente, R. M., GarcíaEscalera, J., \& Chorot, P. (2020). Psychological impact of the COVID19 pandemic: Negative and positive effects in Spanish people during the mandatory national quarantine. Revista de Psicopatologia y Psicologia Clinica, 25(1), 1-22. https://doi.org/10.5944/RPPC.27569

Shah, S. M. A., Mohammad, D., Qureshi, M. F. H., Abbas, M. Z., \& Aleem, S. (2021). Prevalence, Psychological Responses and Associated Correlates 
of Depression, Anxiety and Stress in a

Global Population, During the

Coronavirus Disease (COVID-19)

Pandemic. Community Mental Health

Journal, $\quad$ 57(1), 101-110.

https://doi.org/10.1007/s10597-020-

00728-y

\section{Contacto}

Jorge Malpartida Gutierrez

jorgemalpartida@unat.edu.pe 